\title{
ON THE PREDICTION OF INCUBATION PERIOD IN WATER DROPLET EROSION OF METALS
}

\author{
Mohamed Elhadi Ibrahim ${ }^{1, *}$ and Mamoun Medraj ${ }^{1}$ \\ ${ }^{1}$ Department of Mechanical, Industrial and Aerospace Engineering, Concordia University, 1455 De Maisonneuve Blvd. W. \\ Montreal, Quebec, Canada, H3G 1M8. \\ * Presenter e-mail: $\underline{\text { mo_bra@encs.concordia.ca }}$
}

\begin{abstract}
Water droplet erosion (WDE) is a phenomenon of material loss caused by the repetitive impact of high speed droplets. WDE constitutes a major concern for several industries such as wind energy and efforts to understand the fundamental causes of the phenomenon and methods to combat it are necessary research directions. Essential to combating WDE phenomenon is the ability to predict its onset. This is because erosion damage begins only after an "incubation period" during which stresses are accumulated to the amount necessary to initiate erosion damages. This work presents a model that predicts the number of impacts necessary to end the incubation period. As a prior step, erosion experiments were performed on several metallic materials of known mechanical properties with the aim to identify the target mechanical properties that control materials' resistance to erosion. Experimental data from the literature as well as from our group's earlier studies has also been analyzed to understand the weightages with which the impact parameters (mainly impact velocity and droplet size) affect the incubation period. The model is then developed to predict the incubation period as a function of impact velocity, droplet size, and properties of the target material. So far, it has been observed that fatigue endurance limit, fracture toughness, hardness, and the elastic modulus are the target properties governing the erosion incubation resistance of metallic materials. It was also found that the resistance to the onset of erosion damage decreases as a function of impact velocity to power 4 . The incubation period model has also been compared with three other models from the literature. This work is a part of an ongoing research and preliminary results obtained so far will be presented. These current findings are essential for the development of a full water droplet erosion model which is the overall objective of this work.
\end{abstract}

